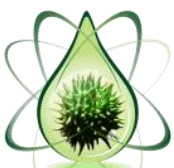

\title{
ESTUDO COMPARATIVO DE MODELOS ESTATÍSTICOS PARA REDUÇÃO DA VISCOSIDADE EM MISTURA DE BIODIESEIS DE MAMONA E SOJA, USANDO UMA METODOLOGIA DE SUPERFÍCIE DE RESPOSTA
}

\author{
Fernanda Mansani da Silva1; Fernanda Rocha Morais'; Gabriel Francisco da Silva1; Ana Eleonora \\ Almeida Paixão ${ }^{1}$ \\ 1 Universidade Federal de Sergipe; fernanda.mansani@gmail.com
}

RESUMO - O biodiesel é um combustível oxigenado produzido a partir da transesterificação de triglicerídeos, presentes em óleos vegetais e gorduras animais. A Agência Nacional do Petróleo, Gás Natural e Biocombustíveis estabele as propriedades a serem usadas para o controle de qualidade do biodiesel, dentre essas, destaca-se a viscosidade cinemática. Seu controle garante um funcionamento adequado dos sistemas de injeção e bombas de combustível. Este trabalho objetiva o estudo comparativo de modelos estatísticos para redução da viscosidade em misturas de biodieseís (Mamona e Soja), usando uma metodologia de superfície de resposta - RSM, baseada em planejamentos fatoriais. Na determinação da viscosidade realizou-se um planejamento fatorial 22 , utilizando como limites de variação mínimos e máximos os seguintes parâmetros: temperaturas $30^{\circ} \mathrm{C}$ e $50^{\circ} \mathrm{C}$ e concentração do biodiesel de referência em relação à mistura $25 \%$ e $75 \%$. Através da RSM, foi possível prever o melhor caminho para se obter um biodiesel com menor viscosidade.

Palavras-chave - Biodiesel, viscosidade, superfície de resposta.

\section{INTRODUÇÃO}

Devido às constantes preocupações ambientais, o biodiesel é uma alternativa interessante como substituição aos combustíveis fósseis, pois seu uso contribui para a diminuição na emissão de $\mathrm{CO}_{2}, \mathrm{SO}$ x e hidrocarboneto aromático durante o processo de combustão (ABREU et al 2004).

O biodiesel é um combustível oxigenado produzido a partir da transesterificação de triglicerídeos, presentes em óleos vegetais e gorduras animais. As propriedades do biodiesel são muito semelhantes às do diesel, porém apresentam uma alta viscosidade, torna o seu uso in natura, como combustível, impraticável.

Diante dessa nova realidade, a Agência Nacional do Petróleo, Gás Natural e Biocombustíveis ANP estabelece as propriedades a serem usadas para o controle de qualidade do biodiesel, bem como 
as especificações e os métodos a serem utilizados. Dentre as propriedades especificadas pela ANP, pode-se destacar a viscosidade cinemática. A viscosidade cinemática, que também é uma propriedade fluidodinâmica, expressa a resistência oferecida pela substância ao escoamento sob gravidade (ASTM D445). $O$ controle da viscosidade de uma substância visa a garantir um funcionamento adequado dos sistemas de injeção e bombas de combustível.

De acordo com a Resolução ANP No 07 de 19.03 .2008 , a viscosidade cinemática a $40^{\circ} \mathrm{C}$ foi limitada à faixa de 3,0 a $6,0 \mathrm{~mm}^{2} / \mathrm{s}$, tornando-se necessário um aprofundamento sobre o estudo dessa propriedade.

O Biodiesel de Mamona possui uma alta viscosidade, por ser proveniente do óleo de Mamona, constituído em sua maioria pelo ácido ricinoleíco. Isto se deve à hidroxila presente na cadeia carbônica que induz à ligação de hidrogênio, ligação forte, intermolecular entre a hidroxila e o oxigênio da carbonila de outro ácido ou a intramolecular, que pode ocorrer na própria molécula entre a hidroxila e a sua carbonila (ANP).

O Biodiesel de Soja é constituído em sua maioria pelo Ácido Linoléico, que possui maior número de ácidos graxos insaturados, ocorrendo uma diminuição da viscosidade, pois há um enfraquecimento da força de dispersão entre as cadeias apolares em decorrência de uma menor área de contato e interações entre as moléculas (ANP).

O presente trabalho consiste numa investigação do comportamento da viscosidade cinemática em misturas de biodieseis de mamona e soja, utilizando-se uma ferramenta estatística denominada Metodologia de Superfície de Resposta (ou RSM) que consiste em uma técnica de otimização baseada em planejamentos fatoriais que foi introduzida por G. E. P. Box nos anos cinquenta, e que desde então tem sido usada com grande sucesso na modelagem de diversos processos industriais.

\title{
METODOLOGIA
}

\section{- VISCOSIDADE CINEMÁTICA}

Materiais:

\author{
Biodiesel de Mamona \\ Biodiesel de Soja
}

Métodos: 
A viscosidade cinemática é determinada em aparelhos denominados viscosímetros. Utilizou-se um Viscosimetro Capilar da Schott-Gerate 150 (Typ 51313), KPG Cannon Fenske, em Banho Termostático.

O viscosímetro cinemático é constituído, basicamente, de um tubo capilar de vidro pelo qual escoa o fluido. $O$ diâmetro do tubo deve ser escolhido de acordo com a viscosidade; para cada tubo é fixado um fator constante "k" para determinar a viscosidade cinemática (v). 0 tempo de escoamento (t) em segundos é anotado quando da passagem do nível do fluido por dois traços de referência no tubo de vidro e calcula-se:

\section{$v=k \cdot t\left(c S t\right.$ ou $\mathrm{mm}^{2} / \mathrm{s}$}

Para o referido viscosímetro; $\mathrm{k}=0,03306 \mathrm{~mm}^{2} / \mathrm{s}$

\section{- PLANEJAMENTO FATORIAL}

O planejamento fatorial constitui uma ferramenta estatística para realização de estudos, sobre um determinado fenômeno, que possuam muitas variáveis, de maneira organizada e com objetivos bem planejados, reduzindo os experimentos a um número mínimo necessário. É usado para estudar como certos fatores influenciam uma determinada resposta, sendo de importância prática.

$\mathrm{O}$ teste $\mathrm{F}$ permite a comparação do valor estimado para $\mathrm{F}$ a partir dos dados experimentais com o valor tabelado para uma distribuição de referência.

\section{- METODOLOGIA DE SUPERFÍCIE DE RESPOSTA (RSM)}

A Metodologia de Superfície de Resposta (ou RSM, de Response Surface Methodology), é uma coleção de técnicas matemáticas e estatísticas usada para a modelagem e análise de problemas em que uma resposta de interesse é influenciada por várias variáveis e o objetivo é otimizar esta resposta.

Esta metodologia tem duas etapas distintas - modelagem e deslocamento - que são repetidas tantas vezes quantas forem necessárias, com o objetivo de atingir uma região ótima da superfície investigada. A modelagem normalmente é feita ajustando-se modelos simples (em geral, lineares ou quadráticos) a respostas obtidas com planejamentos fatoriais. $O$ deslocamento se dá sempre ao longo 
do caminho de máxima inclinação de um determinado modelo, que é a trajetória na qual a resposta varia de forma mais pronunciada (BARROS NETO, 1995).

\section{RESULTADOS E DISCUSSÃO}

\section{VISCOSIDADE CINEMÁTICA UTILIZANDO A METODOLOGIA DE SUPERFÍCIE DE RESPOSTA.}

\section{Modelo Linear:}

Inicialmente, foi realizado um planejamento com apenas um ponto central (valores médios nos intervalos investigados), onde são varridos três níveis de cada fator e não apenas dois, permitindo a verificação da existência ou não da falta de ajuste para um modelo linear que será obtido.

Para o preparo dos blends de biodieseis foi efetuado um planejamento fatorial $2^{2}$ a qual estuda o efeito da temperatura e da concentração do biodiesel de referência (Biodiesel de Mamona) em relação à mistura (Biodieseis de Mamona e Soja) a serem utilizadas.

A matriz de planejamento apresentada na tabela 1, sobre a mistura de biodieseis de mamona e soja, possibilitou a elaboração de uma representação gráfica que é a superfície de resposta e o cálculo dos efeitos dos fatores manipulados sobre a resposta investigada.

As variáveis $\mathrm{x}_{1}$ e $\mathrm{x}_{2}$ representam os valores dos dois fatores codificados pelas equações:

$x_{1}=(T-40) / 10$ (Equação 2) e $x_{2}=(C-50) / 25$ (Equação 3).

O efeito dos fatores manipulados sobre a resposta investigada, indica a magnitude de variação da resposta (em $\mathrm{mm}^{2} / \mathrm{s}$ ) de acordo com a variação dos niveis ( + ou - ) dos fatores, como mostra a tabela 2.

Analisando o teste t, têm-se a equação 4. Verifica-se que que os valores absolutos dos efeitos, inclusive 0 de interação, são superiores a $t_{4} \times \mathrm{s}$ (efeito), portanto, os mesmos são estatisticamente significativos, com 95\% de confiança, na mistura analisada.

$$
t_{4} \times s \text { (efeito) }=2,776 \times 0,108972=0,302506 \mathrm{~mm}^{2} / \mathrm{s}
$$


Através do planejamento experimental, foi obtido o seguinte modelo linear, descrito pela equação 5.

$$
y=7,917-2,18 x_{1}+2,473 x_{2}-0,82 x_{1} x_{2}
$$

A análise de variância (Tabelas 3) traz dados a respeito da significância estatística deste modelo linear.

Como o $F_{R c a l}=M Q_{R} / M Q_{r}$, deve ser maior do que o $F_{R}$, verifica-se que comparando esse valor com $F_{3,3}=9,28$ (ao nível de $95 \%$ ), esse valor indicaria uma regressão significativa, não fosse a evidência de falta de ajuste, devido ao alto valor de $F_{F a j}=M Q_{f a j_{j}} M Q_{e p}$, que é muito maior do que $\circ F_{1,2}=$ 18,51

A superfície de resposta e as curvas de nível (Figuras 1 e 2) para o modelo linear indicam que com o aumento da temperatura há uma redução da viscosidade, e com o aumento da concentração do Biodiesel de Mamona, a viscosidade tende a aumentar, sugerindo o melhor caminho para se obter uma mistura de biodiesel com menor viscosidade.

Apesar da redução da viscosidade cinemática, obtido com o auxilio do modelo linear, a falta de ajuste do modelo não pode ser desconsiderada. Sendo assim, partiu-se para um modelo quadrático.

\section{Modelo Quadrático}

Para isto, é necessário que sejam considerado todos os pontos centrais e os pontos axiais, que é o planejamento em estrela.

A partir dos resultados do planejamento experimental se obteve o modelo quadrático, de acordo com a equação 6 .

$$
y=7,503-1,916 x_{1}+2,377+0,072 x_{1}^{2}+0,557 x_{2}^{2}-0,823 x_{1} x_{2}
$$

A superfície de resposta e as curvas de nível (Figuras 3 e 4) indicam que com o aumento da temperatura há uma redução da viscosidade, assim como com 0 aumento da concentração do Biodiesel de Mamona, a viscosidade tende a aumentar.

A análise de variância (Tabela 5) traz dados a respeito da significância estatística do modelo quadrático. 
Como o $F_{R c a l}=M Q_{R} / M Q_{r}$, deve ser maior do que o $F_{R}$, verifica-se que comparando esse valor com $F_{5,5}=5,05$ (ao nível de 95\%), esse valor indicaria uma regressão significativa, não fosse a evidência de falta de ajuste, devido ao alto valor de $F_{\text {Fajcal }}=M Q_{\text {faj }} / M Q_{\text {ep }}$ que é muito maior do que $\circ F_{3,2}$ $=19,16$.

De acordo com a análise de variância, em relação à porcentagem de variação explicada, que consiste no quociente SQR/SQT,o modelo quadrático que apresentou $99.11844 \%$ de variação explicada está melhor ajustado às respostas em comparação ao modelo linear que apresentou, por sua vez, uma porcentagem de variação explicada de 98,078895\%.

De acordo com a Resolução ANP N 07 de 19/03/2008, que limita a viscosidade cinemática a $40^{\circ} \mathrm{C}$, de 3,0 a $6,0 \mathrm{~mm}^{2} / \mathrm{s}$ apenas o ensaio 11 Mistura de Biodieseis de $15 \%$ de Mamona e $85 \%$ de Soja à temperatura de $40 \mathrm{C}^{\circ}$, se enquadra nessa especificação.

\section{CONCLUSÕES}

Foi de grande importância a elaboração do planejamento fatorial, pois se pode prever o procedimento experimental, servindo como base para o uso da Metodologia de Superfície de Resposta;

De acordo com a Resolução ANP Nº 07 de 19.03.2008, que limita a viscosidade cinemática a $40^{\circ}$, de 3,0 a 6,0 mm²/s, apenas a mistura de Biodieseis de Mamona e Soja, com 15\% de Biodiesel de mamona, se enquadra nessa especificação;

As misturas dos Biodieseis Mamona e Soja consagram-se como uma boa alternativa para a diminuição da viscosidade, porém deve-se utilizar uma pequena proporção do Biodiesel de Mamona;

O modelo quadrático se ajusta melhor às respostas obtidas experimentalmente (de acordo com a ANOVA), em relação ao modelo linear na mistura estudada.

\section{REFERÊNCIAS}

ABREU, F. R.; LIMA, D. G.; HAMÚ, E. H.; WOLF,C.;SUAREZ, P. A. Z. Utilization of metal complexes as catalysts in the transesterification of Brazilian vegetable oils with different alcohols, Journal of Molecular Catalysis, 29: 209, 2004.

ANP, Agência de Petróleo, Gás Natural e Biocombustíveis. Resolução Nº 07 de 19.03.2008. 
ASTM D 445. Standard Test Method for Kinematic Viscosity of Transparent and Opaque Liquids (the Calculation of Dynamic Viscosity). Annual Book of ASTM Standards, Vol. 05.01, p. 185193, 2001.

BARROS NETO, B.; SCARMINIO, I. S.; BRUNS, R. E. Planejamento e Otimização de Experimentos. Campinas: Editora da Unicamp, 299p., 1995.

BOX, G. E. P.; WILSON, K. B.; J. Roy. Statist. Soc., Ser. B 1951, 13, 1.

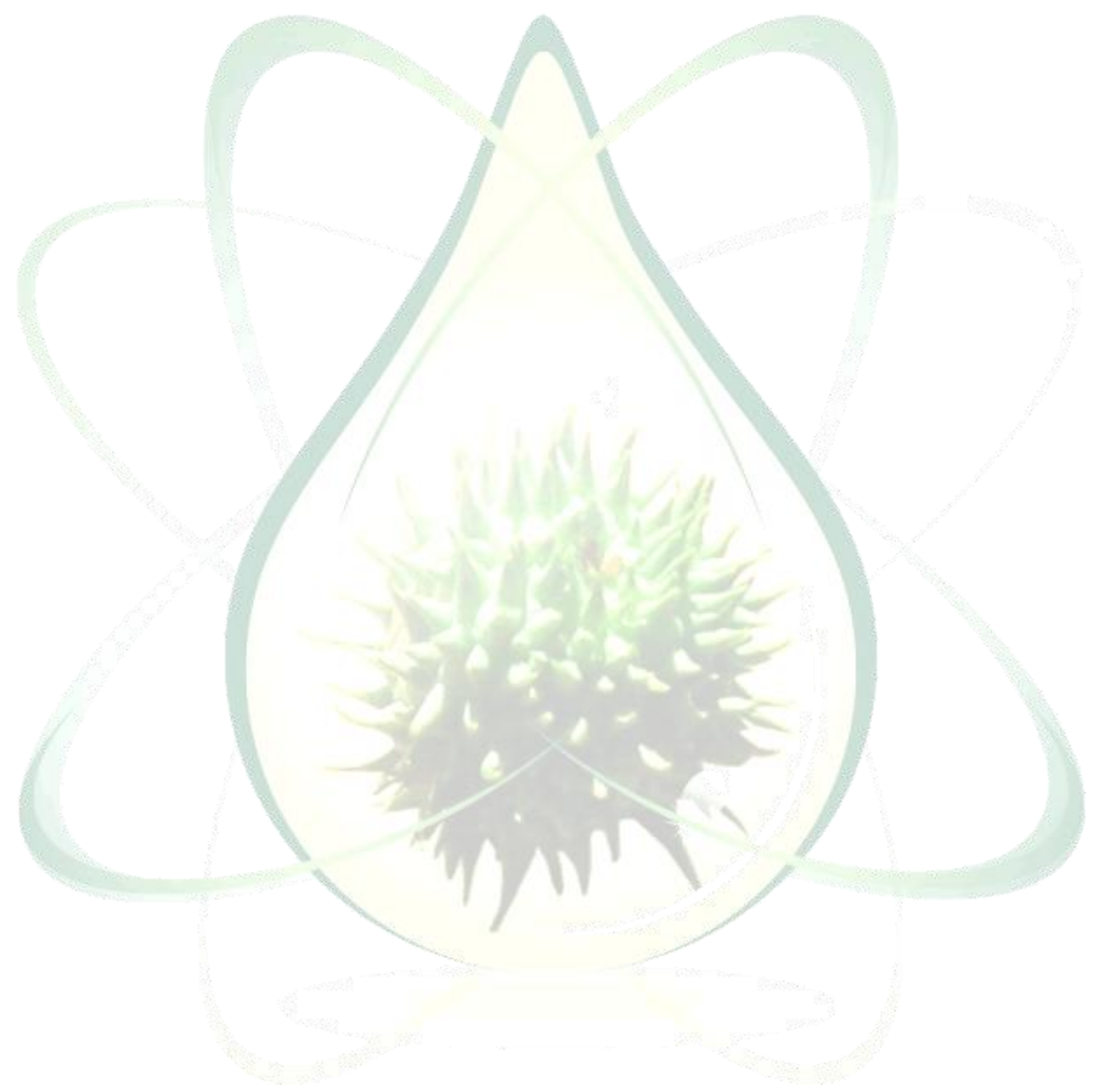


Tabela 2. Matriz de Planejamento para a Mistura de Biodieseis de Mamona e Soja.

\begin{tabular}{cccccc}
\hline Ensaio & $\begin{array}{c}\mathrm{T} \\
\left({ }^{\circ} \mathrm{C}\right)\end{array}$ & $\begin{array}{c}\mathrm{C} \\
(\%)\end{array}$ & $\mathbf{x}_{1}$ & $\mathbf{x}_{2}$ & $\begin{array}{c}\mathrm{vm} \\
\left(\mathrm{mm}^{2} / \mathbf{s}\right)\end{array}$ \\
\hline 1 & 30 & 25 & -1 & -1 & 7,11 \\
2 & 50 & 25 & 1 & -1 & 4,40 \\
3 & 30 & 75 & -1 & 1 & 13,70 \\
4 & 50 & 75 & 1 & 1 & 7,70 \\
5 & 40 & 50 & 0 & 0 & 7,50 \\
\hline
\end{tabular}

Tabela 3. Efeitos dos fatores calculados para o planejamento fatorial $2^{2}$ da Tabela 1.

\begin{tabular}{ll}
\hline Média global & $8,23 \pm 0,038528$ \\
\hline Efeitos principais: & $-4,355 \pm 0,108972$ \\
$E_{1}$ & $4,945 \pm 0,108972$ \\
$E_{2}$ & \\
Efeito interação & $-1,645 \pm 0,108972$ \\
$E_{12}$ & \\
\hline
\end{tabular}

Tabela 4. Análise da Variância (ANOVA) para a mistura de Biodieseis Mamona e Soja, considerando modelo linear.

\begin{tabular}{l|ccc|c}
\hline $\begin{array}{l}\text { Fonte } \\
\text { de Variação }\end{array}$ & SQ & MQ & G.L & Fcal \\
\hline Regressão $(\mathrm{R})$ & 46,1 & 15,4 & 3 & 51,053 \\
\hline Resíduo $(\mathrm{r})$ & 0,9 & 0,3 & 3 & \\
\hline Falta de ajuste $\left(\mathrm{F}_{\mathrm{aj}}\right)$ & 0,9 & 0,9 & 1 & 402,436 \\
\hline Erro puro (ep) & 0 & 0 & 2 & \\
\hline Total & 47 & & 6 & \\
\hline
\end{tabular}

\% variação explicada: $\mathbf{9 8 , 0 7 8 8 9 5}$

\% máx. de variação explicável: 99,990502 
Tabela 5. Matriz do planejamento em estrela para a Mistura de Biodieseis Mamona e Soja.

\begin{tabular}{cccccc}
\hline Ensaio & $\begin{array}{c}\text { Temperatura } \\
\left({ }^{\circ} \mathbf{C}\right)\end{array}$ & $\begin{array}{c}\text { Concentração } \\
(\%)\end{array}$ & $\mathbf{x}_{\mathbf{1}}$ & $\mathbf{x}_{\mathbf{2}}$ & $\mathbf{v}_{\mathbf{m}}\left(\mathbf{m m}^{2} / \mathbf{s}\right)$ \\
\hline 1 & 30 & 25 & -1 & -1 & \\
2 & 50 & 25 & 1 & -1 & 7,11 \\
3 & 30 & 75 & -1 & 1 & 4,40 \\
4 & 50 & 75 & 1 & 1 & 13,70 \\
5 & 40 & 50 & 0 & 0 & 7,70 \\
6 & 40 & 50 & 0 & 0 & 7,45 \\
7 & 40 & 50 & 0 & 0 & 7,54 \\
8 & 26 & 50 & $-1,41$ & 0 & 7,52 \\
9 & 40 & 85 & 0 & 1,41 & 9,88 \\
10 & 54 & 50 & 1,41 & 0 & 11,73 \\
11 & 40 & 15 & 0 & $-1,41$ & 5,22 \\
\hline
\end{tabular}

Tabela 6. Análise de variância para a Mistura de Biodieseis Mamona e Soja, considerando modelo quadrático.

\begin{tabular}{clllll}
\hline & $\begin{array}{l}\text { Fonte } \\
\text { de Variação }\end{array}$ & SQ & MQ & G.L & Fcal \\
\hline (R) & Regressão & 78,8230 & 15,7650 & 5 & 112,435 \\
\hline & Resíduo (r) & 0,7010 & 0,1402 & 5 & \\
\hline $\begin{array}{c}\text { Falta de } \\
\text { ajuste (Faj) }\end{array}$ & 0,6970 & 0,2322 & 3 & 103,968 \\
\hline & Erro puro & 0,0045 & 0,0045 & 2 & \\
(ep) & Total & 79,5250 & & 10 & \\
\hline
\end{tabular}

\% variação explicada: 99,11844

\% máx. de variação explicável: 

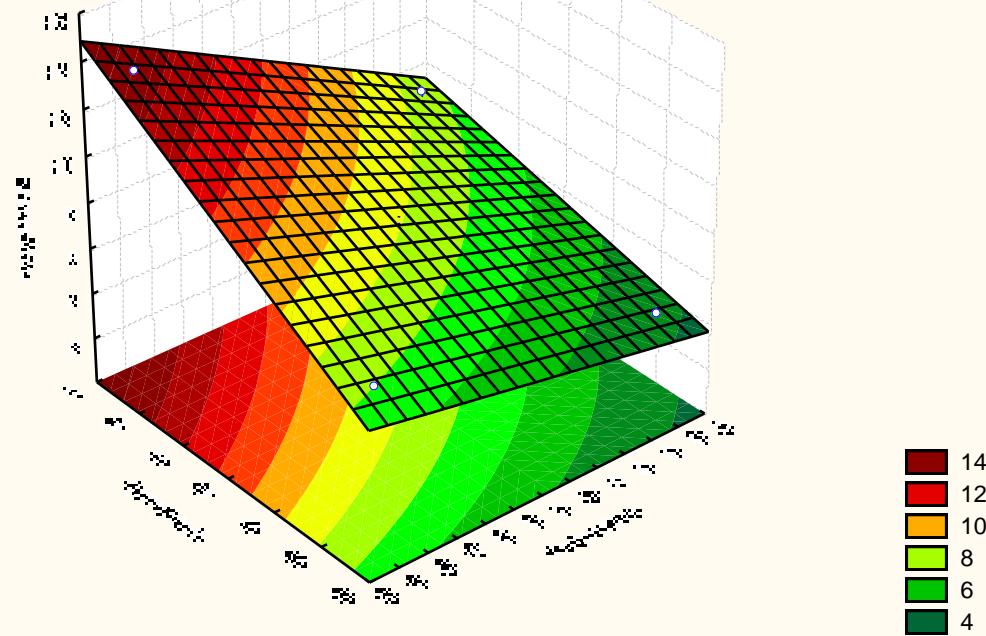

Figura 4. Superfície de Resposta para a Mistura de Biodieseis de Mamona e Soja, considerando modelo linear.

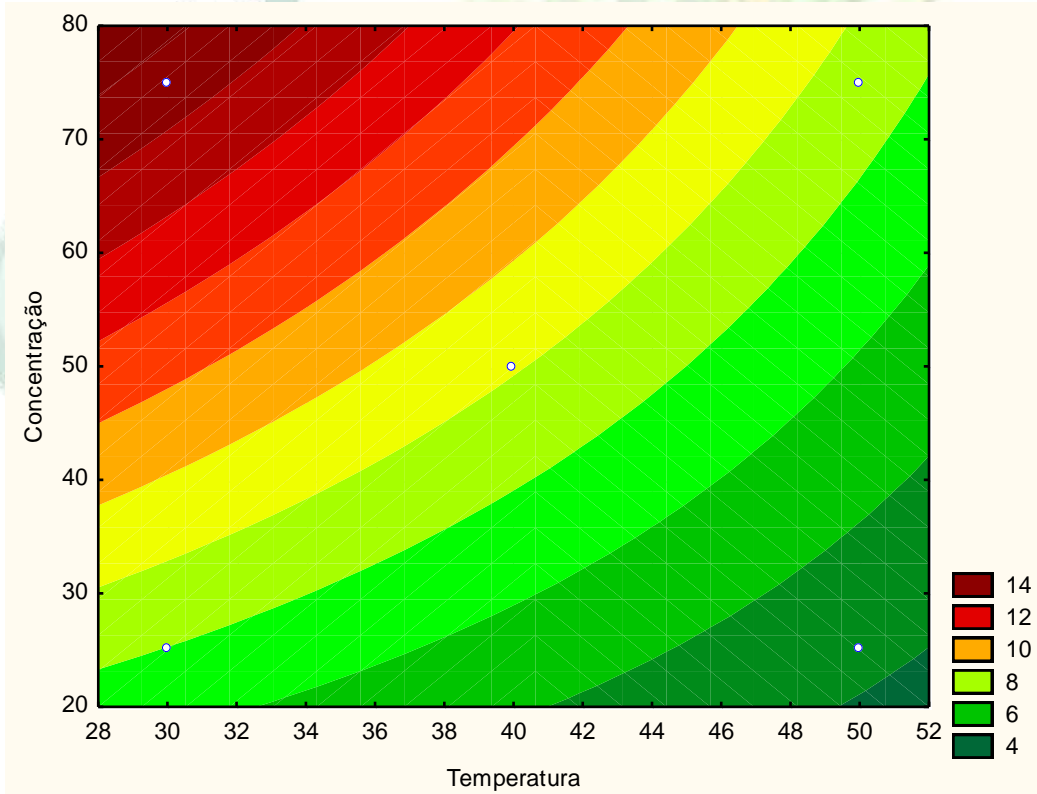

Figura 5. Curvas de Nível do plano descrito pela Equação linear. 

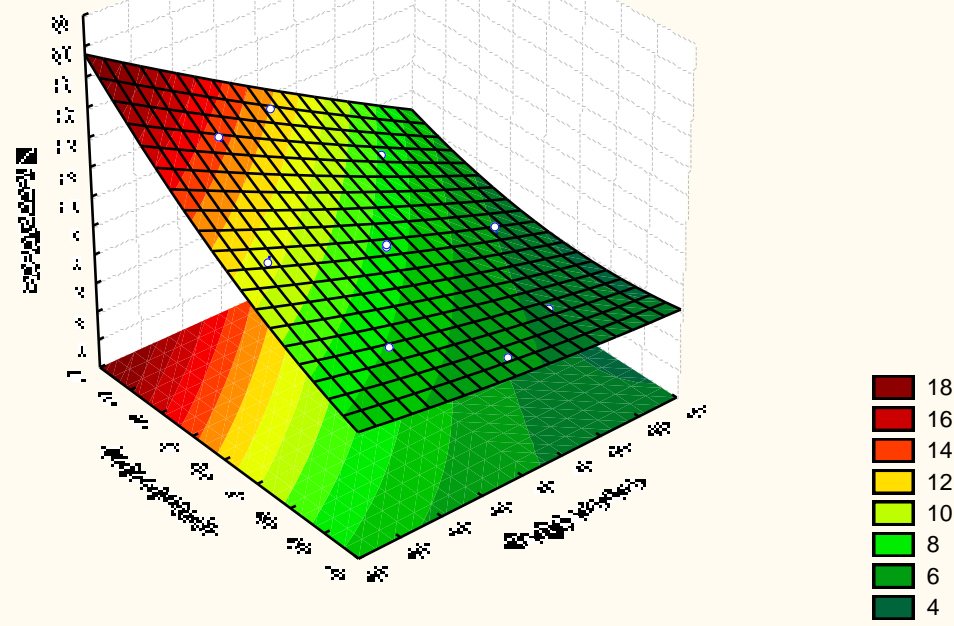

Figura 6. Superfície de Resposta para a Mistura de Biodieseis de Mamona e Soja, considerando modelo quadrático.

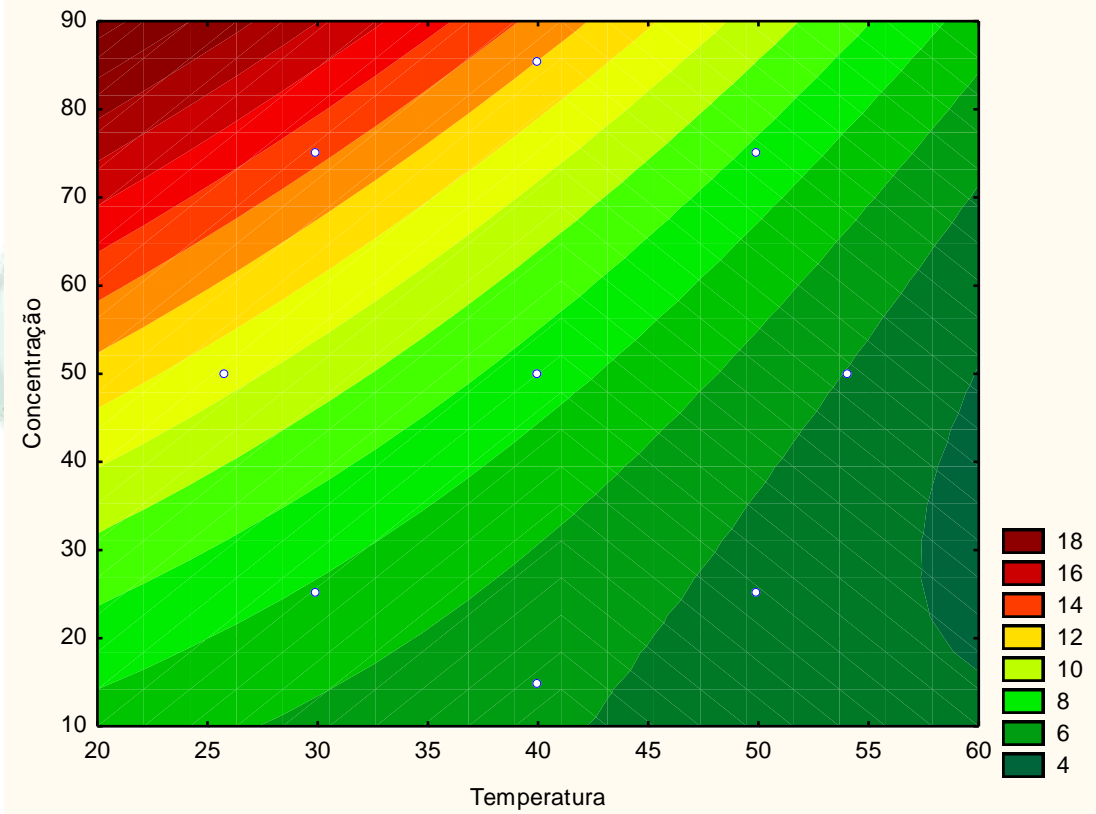

Figura 7. Curvas de Nível do plano descrito pela Equação quadrática. 